\title{
Contribution to the Theory of Frenkel Excitons in Disordered Molecular Crystals*
}

\author{
Hwei-Kwan Hong and RaOul Kopelman \\ Department of Chemistry, University of Michigan, Ann Arbor, Michigan 48104
}

(Received 18 May 1971)

\begin{abstract}
In this paper, we discuss the Frenkel excitons in disordered molecular crystals. Most of our results can be generalized to cover electrons and phonons in other disordered systems as well. In particular, the relationship among the exact Green's function, the CPA (coherent potential approximation) Green's function, and the method of moments is discussed. It is emphasized that, despite its complicated form, the exact Green's function not only yields correct moments for the spectral density function and the overall density-of-states function but also leads to the CPA Green's function if certain terms are modified. Both the exact and the CPA self-energies are expanded with the aid of generating functions. This enables us to demonstrate both systemmatically and analytically how the latter is derived from the former and what exactly the approximations are. A better understanding of the CPA is thus obtained. Specifically, it is shown that the exact self-energy is wave vector and branch independent in the first five terms of its expansion in powers of $Z^{-1}$ (reciprocal energy). A comparison with a similar expansion of the CPA selfenergy indicates that the CPA self-energy is exact to the $Z^{-5}$ power. Consequently, the spectral density function and the over-all density-of-states functions determined from the CPA method have the correct seven and eight lower moments, respectively. Analytical expressions for these lower moments are also given for future applications.
\end{abstract}

\section{INTRODUCTION}

Perfect solids are characterized by the translational invariance of the lattice. When such invariance is destroyed by the introduction of foreign atoms or molecules, a disordered system is formed, the physicalchemical-biological properties of which can no longer be handled with the help of group theory. In the last decade, various techniques ${ }^{1}$ have been devised and applied to the electronic (or phonon) states of disordered systems. These include the moment trace method, ${ }^{2}$ the negative factor counting method, ${ }^{3}$ the phase theory, ${ }^{4}$ and last, but not least, the Green's function method. ${ }^{5}$

Formally, a complete analysis of disordered systems is possible through the application of the Green's function method. Following the pioneering work of $\mathrm{Lax}^{5}$ on the multiple scattering formalism, Yonezawa and Matsubara ${ }^{6}$ recently carried out a most extensive analysis of the random lattice problem, using the Green's function method. They obtained a complete expansion of the disorder-perturbed Green's function which contained, in principle, all the information of a disordered binary system with arbitrary composition and energy separation. Due to difficulties involved in the factoring of the expansion terms, a closed form proved to be inaccessible. Rather, Onodera and Toyozawa ${ }^{7}$ attempted to use an approximation which really amounted to assuming a $\mathbf{k}$-independent self-energy in a model calculation on electrons and excitons. Such approximation was also independently proposed by Taylor ${ }^{8}$ for phonons and by Soven ${ }^{9}$ for alloys, the latter called it the Coherent Potential Approximation (CPA). In recent papers by Velicky et al..$^{10}$ and by Soven, ${ }^{11}$ the validity and the range of applicability of such an approximation were further exposed.

In the related field of Frenkel-Davydov excitons in disordered molecular crystals, which frequently con- tain more than one molecule per unit cell, Hong and Robinson ${ }^{12 a}$ have extended Yonezawa and Matsubara's formulation to such systems involving multiplebranched exciton bands. Within the limit of the restricted Frenkel exciton case, ${ }^{13}$ they have shown that the statistical averaging can be performed to obtain the exact mixed-crystal Green's function. Furthermore, in the spirit of CPA, the mixed-crystal density-ofstates functions and the optical spectra can be calculated from the following information: (i) The over-all density-of-states function of the corresponding pure crystal (including all the branches) and (ii) the location of all the Davydov components (the $\mathbf{k}=\mathbf{0}$ states). This theory was applied to the ${ }^{1} B_{2 u}$ exciton states of heavily doped isotopic mixed crystals of naphthalene. Using the pure crystal density-of-states function obtained experimentally from the hot-band spectroscopy ${ }^{14}$ and the known Davydov components of naphthalene, they have satisfactorily accounted for most of the features observed in a parallel experimental study. ${ }^{12 b}$

In this paper, we shall deal specifically with restricted Frenkel-Davydov excitons in heavily doped mixed crystals, although, as will be seen, most of our results and conclusions are valid for electrons and phonons in simple lattices as well. First, the relationship between the Green's function and the moments is discussed. In doing so, we attempt to show the consistency between the exact self-energy obtained by Hong and Robinson ${ }^{12}$ and the method of moments. It is stressed that, while we can always obtain moments from the exact Green's function, the reverse is apparently not true. Second, higher expansion terms of the exact self-energy are obtained, using the recipe by Velicky et al. It is found that the expansion of the exact self-energy is $\mathbf{k}$ independent and branch independent up to $Z^{-5}$ exactly. The first eight moments of the spectral density and those of the over-all density-of-states function are calculated (to be compared later on with our computer-simulated 
results). A comparison with a similar expansion of the CPA self-energy leads to the conclusion that, for the over-all density-of-states function, the CPA results are correct up to (and including) the seventh moment. On the other hand, the CPA optical spectra are correct only up to (and including) the sixth moment. Finally, the relationship between the exact self-energy and the CPA self-energy is discussed from a more analytical point of view. This is done by comparing the various scattering routes (or diagrams) involved in both cases and exploring the relationships among them. A transformation of parameter from concentration to $\omega\left(=C_{B}-C_{A}\right)$ facilitates the expansion of the CPA selfenergy and its comparison to the exact self-energy.

We have attempted in this paper to lay down some of the theoretical background necessary for a later publication ${ }^{15}$ on random lattice calculations. It is hoped that these investigations will add to our understanding of the electronic states in heavily doped mixed molecular crystals in particular and those of disordered systems in general.

\section{THE EXACT GREEN'S FUNCTION AND MOMENTS}

Among the various techniques that have been developed for disordered systems, the phase theory stood out as the only technique that dealt directly with eigenfunctions. However, it was exactly this fundamental way of approach that prohibited its adaption for twoand three-dimensional systems. Since the numerical technique of Dean $^{3}$ will be discussed in a later publication from this laboratory, ${ }^{15}$ we should discuss here the Green's function method and the method of moments as applied to heavily doped mixed molecular crystals and other more general aspects.

One of the earlier attempts to tackle the problem of disordered solids was made by Domb et al..$^{2}$ who tried the method of moments (or the moment trace method). Since the moments of the density-of-states function can be evaluated analytically, a method was proposed by Domb et al., ${ }^{2}$ which involved the expansion of the density-of-states function in terms of polynomials such as Legendre polynomials and subsequent determination of expansion coefficients from the known lower moments (all told, 20 moments were used in calculations by Domb et al.). Due to the fact that smooth, wellbehaved polynomials were used, the resulting densityof-states function failed to bear out the irregular nature of the exact solutions. ${ }^{16}$ It would seem that moments can best be used to check the results based on other methods rather than to evaluate the actual distribution of eigenvalues.

An alternative approach which has recently gained a considerable amount of popularity is the Green's function method. For molecular crystals of nontrivial interchange symmetries ${ }^{17}$ Hong and Robinson ${ }^{12}$ have shown that, within the restricted Frenkel-Davydov limit, the exact self-energies (and hence the Green's function) can be diagonalized with the basis set that also diagonalizes the pure crystal Hamiltonian. This is due to the fact that crystal symmetries "appear" to be restored when impurities are randomly situated. For example, for molecular crystals with an interchange group of order two (such as naphthalene) the basis set is:

$$
|\mathbf{k}, j\rangle=N^{-1 / 2}\left[\sum_{\alpha} \exp \left(i \mathbf{k} \cdot \mathbf{R}_{\alpha}\right)-(-1)^{j} \sum_{\beta} \exp \left(i \mathbf{k} \cdot \mathbf{R}_{\boldsymbol{\beta}}\right)\right]
$$

where $j=1,2$ designate the two branches (note the relation to Ref. $12:|\mathbf{k}, 1\rangle=\left|\mathbf{k}^{+}\right\rangle$and $\left.|\mathbf{k}, 2\rangle=\left|\mathbf{k}^{-}\right\rangle\right)$ and $R_{\alpha}$ and $R_{\beta}$ are the displacement vectors for translationally equivalent and interchange equivalent molecules, respectively. Since interchange symmetries were incorporated into the wavefunctions of all the $\mathbf{k}$ states, the selection rules for the exciton-impurity scattering can be simply decomposed into two parts, one associated with the translational symmetry of the crystal and the other associated with one of the interchange symmetries of the crystal. For nonsymmorphic (or symmorphic, with more than one molecule per primitive unit cell) crystals, this decomposition is only valid in case of short-range interactions. ${ }^{18}$

The exciton self-energy was obtained by Hong and Robinson ${ }^{12 a}$ as:

$$
\begin{aligned}
\Sigma(\mathbf{k}, j)=\Delta^{2} P_{\mathbf{2}}\left(C_{B}\right) \sum_{j^{\prime}} \sum_{k^{\prime}} N^{-1}\left\langle G\left(\mathbf{k}^{\prime}, j^{\prime}\right)\right\rangle+\Delta^{3} P_{3}\left(C_{B}\right) \sum_{j^{\prime}} \sum_{j^{\prime \prime}} \sum_{k^{\prime}} \sum_{k^{\prime \prime}} N^{-2}\left\langle G\left(\mathbf{k}^{\prime}, j^{\prime}\right)\right\rangle\left\langle G\left(\mathbf{k}^{\prime \prime}, j^{\prime \prime}\right)\right\rangle \\
+\Delta^{4} P_{4}\left(C_{B}\right) \sum_{j^{\prime}} \sum_{j^{\prime \prime}} \sum_{j^{\prime \prime \prime}} \sum_{k^{\prime}} \sum_{k^{\prime \prime}} \sum_{k^{\prime \prime \prime}} N^{-3}\left\langle G\left(\mathbf{k}^{\prime}, j^{\prime}\right)\right\rangle\left\langle G\left(\mathbf{k}^{\prime \prime}, j^{\prime \prime}\right)\right\rangle\left\langle G\left(\mathbf{k}^{\prime \prime \prime}, j^{\prime \prime \prime}\right)\right\rangle \\
+\Delta^{4} P_{2}{ }^{2}\left(C_{B}\right) \sum_{j^{\prime}} \sum_{j^{\prime \prime}} \sum_{j^{\prime \prime \prime}} \sum_{k^{\prime}} \sum_{k^{\prime \prime}} \sum_{k^{\prime \prime \prime}} N^{-2} \bar{\delta}\left(\mathbf{k}-\mathbf{k}^{\prime}+\mathbf{k}^{\prime \prime}-\mathbf{k}^{\prime \prime \prime}\right)\left\langle G\left(\mathbf{k}^{\prime}, j\right)\right\rangle\left\langle G\left(\mathbf{k}^{\prime \prime} j^{\prime \prime}\right)\right\rangle\left\langle G\left(\mathbf{k}^{\prime \prime \prime}, j^{\prime \prime \prime}\right)\right\rangle+\cdots
\end{aligned}
$$

ind

$$
\langle G(\mathbf{k}, j)\rangle \equiv[E-\epsilon-\epsilon(\mathbf{k}, j)-\Sigma(\mathbf{k}, j)]^{-\mathbf{1}},
$$

where $\Delta \equiv \epsilon_{B}-\epsilon_{A}$ and $Y$ is the total number of molecules. $\epsilon \equiv C_{A} \epsilon_{A}+C_{B} \epsilon_{B}$ is the weighted mean of excitation energies $\epsilon_{A}$ and $\epsilon_{B}$. Component $A$ was taken to be the major constituent. We have explicitly included $\sum_{j}$ to 
denote the summation over all branches. Notice that $\boldsymbol{\epsilon}(\mathbf{k}, 1)=\epsilon\left(\mathbf{k}^{+}\right)$and $\epsilon(\mathbf{k}, 2)=\boldsymbol{\epsilon}\left(\mathbf{k}^{-}\right)$, where

$$
\begin{aligned}
& \epsilon\left(\mathbf{k}^{+}\right)=I_{\alpha \alpha}(\mathbf{k})+I_{\alpha \beta}(\mathbf{k}), \\
& \epsilon\left(\mathbf{k}^{-}\right)=I_{\alpha \alpha}(\mathbf{k})-I_{\alpha \beta}(\mathbf{k}) .
\end{aligned}
$$

Here $I_{\alpha \alpha}$ and $I_{\alpha \beta}$ are the modulated sums of translationally equivalent and interchange equivalent interactions, respectively. ${ }^{19}$ The $P_{n}\left(C_{B}\right)$ are polynomials obtained by Yonezawa and Matsubara. ${ }^{6}$ They are, indeed, the probability functions for different scattering routes. For reasons which will become clear later, we have rewritten these polynomials as $Y_{l}(\omega)$, where $\omega=C_{B}-C_{A}$, as shown in Appendix A. The $\bar{\delta}$ can be considered as representing the selection rules. Mathematically it can be conveniently written as ${ }^{12,20}$

$$
\bar{\delta}(\mathbf{p})=\delta(\mathbf{p}) H\left[(-1)^{m}\right],
$$

where $H\left[(-1)^{m}\right]$ is the Heaviside function and $m$ is the number of times excitons are scattered from one branch to the other. A diagrammatic representation ${ }^{12,21}$ of Eq. (2) is shown in Fig. 1(a). It can be seen that the propagator $\langle G(\mathbf{k}, j)\rangle$ is represented as a thick horizontal line and impurities as vertices. Each vertex is associated with a polynomial $P_{n}\left(C_{B}\right)$ or, equivalently, $Y_{n}(\omega)$ where $n$ is the number of interaction lines connecting the vertex and the propagator. Notice that the angular bracket in Eqs. (2) and (3) indicates that the average Green's function is obtained by averaging over all possible configurations of impurity distributions. On the contrary, only one such configuration is usually treated in the method of negative factor counting. ${ }^{3}$ The latter method apparently converges to the former if the number of molecules treated becomes very large.

To sum the expansions and get a closed form prove to be very difficult, although in certain limits, certain partial summations can be performed. ${ }^{20}$ However, important information can be obtained from the exact self-energy. One set of quantities which are readily obtainable are various moments of the spectral density $a(\mathbf{k}, j)$ and those of the over-all density of states func-

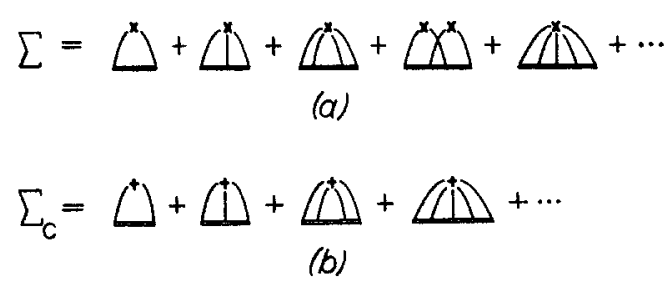

FIG. 1. (a) Diagrammatic representation of the expansion of the exact self-energy $\Sigma(k, j)$ in terms of the exact Green's function $\langle G(\mathbf{k}, j)\rangle$. See text. (b) Diagrammatic representation of the expansion of the CPA self-energy $\Sigma_{e}(E)$ in terms of the CPA Green's function $\langle G(\mathbf{k}, j)\rangle_{c}$. See text. tion $\rho(E)$. Using the notation of Velicky et al., ${ }^{10}$ we define

$$
\begin{aligned}
a(\mathbf{k}, j) & =\pi^{-1} \operatorname{Im}\langle G(\mathbf{k}, j)\rangle \\
& =\sum_{n}|\langle n \mid \mathbf{k}, j\rangle|^{2} \delta\left(E-E_{n}\right), \\
\rho(E) & =N^{-1} \sum_{j} \sum_{k} a(\mathbf{k}, j)=(N \pi)^{-1} \operatorname{Im}[\operatorname{Tr}\langle G(\mathbf{k}, j)\rangle] \\
& =V^{-1} \sum_{n} \delta\left(E-E_{n}\right),
\end{aligned}
$$

where $|n\rangle$ is an eigenstate of the mixed crystal and $E_{n}$ is its eigenvalue. It is understood that we have to substitute $Z \equiv E-i 0^{+}$into $\mathrm{Eq}$. (3) to obtain Eq. (6a). Here $0^{+}$is a small positive quantity. Also notice that $a(\mathbf{0}, 1)$ and $a(\mathbf{0}, 2)$ are simply the optical spectra (polarized parallel and perpendicular to the $\mathbf{b}$ axis, respectively, for naphthalene). The moments of $a(\mathbf{k}, j)$ and $\rho(E)$ are defined as:

$$
\begin{gathered}
M_{p}(\mathbf{k}, j)=\int a(\mathbf{k}, j) E^{p} d E \\
\mu_{p}(E)=\int \rho(E) E^{p} d E .
\end{gathered}
$$

They are related by the following expression:

$$
\mu_{p}(E)=N^{-1} \sum_{j} \sum_{k} M_{p}(\mathbf{k}, j) .
$$

It is well-known ${ }^{10,22}$ that moments are related to the expansion coefficients in an expansion of the true Green's function in powers of $Z^{-1}$. We have

$\langle G(\mathbf{k}, j)\rangle=Z^{-1}+M_{1}(\mathbf{k}, j) Z^{-2}+M_{2}(\mathbf{k}, j) Z^{-3}+\cdots$.

Knowing the exact self-energy [Eq. (2)], and hence the exact Green's function [Eq. (3)], we can evaluate $M_{p}(\mathbf{k}, j)$ and, from Eq. (7c), $\mu_{p}(E)$. This can be most conveniently done by first expanding $\langle G(\mathbf{k}, j)\rangle$ in Eq. (3) :

$$
\begin{aligned}
\langle G(\mathbf{k}, j)\rangle=Z^{-1} & +[\epsilon+\epsilon(\mathbf{k}, j)+\Sigma(\mathbf{k}, j)] Z^{-2} \\
+ & {[\epsilon+\epsilon(\mathbf{k}, j)+\Sigma(\mathbf{k}, j)]^{2} Z^{-3}+\cdots }
\end{aligned}
$$

A similar expansion of $\boldsymbol{\Sigma}(\mathbf{k}, j)$ takes the form of

$$
\Sigma(\mathbf{k}, j)=\sum_{p=1}^{\infty} \Lambda_{p} Z^{-p} .
$$

Equations (9) and (10) are both substituted into Eq. (2). By comparing the coefficients on both sides, $\Lambda_{p}$ 's are determined. This process is straightforward al though it is somewhat cumbersome. The final expressions for $\Lambda_{p}$ 's are more compact, if the zero of the energy scale is placed ${ }^{10}$ at $\frac{1}{2}\left(\epsilon_{A}+\epsilon_{B}\right)$. Thus we have $\epsilon_{A}=-\Delta / 2$, $\epsilon_{B}=\Delta / 2$, and $\epsilon=\left(C_{B}-C_{A}\right) \Delta / 2 \equiv \omega \Delta / 2$. The first six 
$\Lambda_{p}$ 's were obtained. Equation (10) then gives:

$$
\begin{gathered}
\Sigma(\mathbf{k}, j)=x y \Delta^{2}\left[Z^{-1}-\epsilon Z^{-2}+\left(\mu_{2}{ }^{(0)}+\epsilon^{2}\right) Z^{-3}\right. \\
+\left(\mu_{3}{ }^{(0)}-\epsilon^{3}-\epsilon \mu_{2}{ }^{(0)}\right) Z^{-4}+\left(\mu_{4}{ }^{(0)}+2 \epsilon^{2} \mu_{2}{ }^{(0)}+\epsilon^{4}\right. \\
\left.+x y \Delta^{2} \mu_{2}{ }^{(0)}\right) Z^{-5}+\left(\mu_{5}{ }^{(0)}-\epsilon^{5}+\epsilon \mu_{4}{ }^{(0)}+\frac{1}{2} \Delta^{2} \mu_{3}{ }^{(0)}\right. \\
\left.\left.\quad-\epsilon^{3} \mu_{2}{ }^{(0)}-\frac{1}{4} \epsilon \Delta^{2} \mu_{2}{ }^{(0)}-2 \epsilon \mu_{2}{ }^{(0)} \mu_{2}{ }^{(0)}\right) Z^{-6}\right] \\
\quad+x y \Delta^{2}\left[\sum_{\alpha} W^{3}\left(\mathbf{R}_{\alpha}\right) \exp \left(i \mathbf{k} \cdot \mathbf{R}_{\alpha}\right)\right. \\
\left.-(-1)^{j} \sum_{\beta} W^{3}\left(\mathbf{R}_{\beta}\right) \exp \left(i \mathbf{k} \cdot \mathbf{R}_{\beta}\right)\right] Z^{-6}+\cdots
\end{gathered}
$$

where $W\left(\mathbf{R}_{\alpha}\right)$ and $W\left(\mathbf{R}_{\beta}\right)$ are translationally and interchange equivalent pairwise interactions, ${ }^{21}$ respectively, and

$$
\mu_{n}{ }^{(0)}=N^{-1} \sum_{j} \sum_{k} \epsilon^{n}(\mathbf{k}, j)
$$

is the $n$th moment of the pure crystal density-of-states function. To conform with the notation of Velicky et al., we also put $C_{A}=x, C_{B}=y$. Our $\Delta$ is defined differently in sign from their $\delta$, but this is of no consequence here since only even powers are involved. It is noted that there are no $\mathbf{k}, j$-dependent terms inside the first bracket. The first $\mathbf{k}, j$-dependent term occurs with $Z^{-6}$, which actually comes from the fourth diagram in Fig. 1(a). Further discussion concerning diagrams of this type is reserved for later sections when the relationship between the CPA Green's function and the exact Green's function will be discussed.

It is now a simple matter to calculate $M_{p}(\mathbf{k}, j)$. Equation (11) is substituted into Eq. (9) and the final expression is compared with Eq. (8). In this way, we find that:

$$
\begin{aligned}
& M_{0}(\mathbf{k}, j)=1 \text {, } \\
& M_{\mathbf{1}}(\mathbf{k}, j)=\epsilon+\epsilon(\mathbf{k}, j) \text {, } \\
& M_{2}(\mathbf{k}, j)=\left(\Delta^{2} / 4\right)+2 \epsilon \cdot \epsilon(\mathbf{k}, j)+\epsilon^{2}(\mathbf{k}, j) \text {, } \\
& M_{3}(\mathbf{k}, j)=\left(\epsilon \Delta^{2} / 4\right)+\left(\Delta^{2} / 2+\epsilon^{2}\right) \cdot \epsilon(\mathbf{k}, j)+3 \epsilon \cdot \epsilon^{2}(\mathbf{k}, j)+\epsilon^{3}(\mathbf{k}, j) \text {, } \\
& M_{4}(\mathbf{k}, j)=\left(\Delta^{4} / 16\right)+x y \Delta^{2} \mu_{2}{ }^{(0)}+\epsilon \Delta^{2} \cdot \epsilon(\mathbf{k}, j)+\left(3 \Delta^{2} / 4+3 \epsilon^{2}\right) \cdot \epsilon^{2}(\mathbf{k}, j)+4 \epsilon \cdot \epsilon^{3}(\mathbf{k}, j)+\epsilon^{4}(\mathbf{k}, j) \text {, } \\
& M_{5}(\mathbf{k}, j)=\left(\epsilon \Delta^{4} / 16\right)+x y \epsilon \Delta^{2} \mu_{2}{ }^{(0)}+x y \Delta^{2} \mu_{3}{ }^{(0)}+\left[\left(3 \Delta^{4} / 16\right)+\left(\epsilon^{2} \Delta^{2} / 2\right)+2 x y \Delta^{2} \mu_{2}{ }^{(0)}\right] \cdot \epsilon(\mathbf{k}, j) \\
& +\left[\left(9 \epsilon \Delta^{2} / 4\right)+\epsilon^{3}\right] \cdot \epsilon^{2}(\mathbf{k}, j)+\left(\Delta^{2}+6 \epsilon^{2}\right) \cdot \epsilon^{3}(\mathbf{k}, j)+5 \epsilon \cdot \epsilon^{4}(\mathbf{k}, j)+\epsilon^{5}(\mathbf{k}, j), \\
& M_{6}(\mathbf{k}, j)=\left(\Delta^{6} / 64\right)+x y \Delta^{2} \mu_{4}{ }^{(0)}+2 \epsilon x y \Delta^{2} \mu_{3}{ }^{(0)}+\left(3 x y \Delta^{4} \mu_{2}{ }^{(0)} / 4\right)+\left[\left(3 \epsilon \Delta^{4} / 8\right)+4 \epsilon x y \Delta^{2} \mu_{2}{ }^{(0)}+2 x y \Delta^{2} \mu_{3}{ }^{(0)}\right] \cdot \epsilon(\mathbf{k}, j) \\
& +\left[\left(3 \Delta^{4} / 8\right)+\left(9 \epsilon^{2} \Delta^{2} / 4\right)+3 x y \Delta^{2} \mu_{2}{ }^{(0)}\right] \cdot \epsilon^{2}(\mathbf{k}, j)+\left(4 \epsilon \Delta^{2}+4 \epsilon^{3}\right) \cdot \epsilon^{3}(\mathbf{k}, j) \\
& +\left[\left(5 \Delta^{2} / 4\right)+10 \epsilon^{2}\right] \cdot \epsilon^{4}(\mathbf{k}, j)+6 \epsilon \cdot \epsilon^{5}(\mathbf{k}, j)+\epsilon^{6}(\mathbf{k}, j), \\
& M_{7}(\mathbf{k}, j)=\left(\epsilon \Delta^{6} / 64\right)+\left(3 \epsilon x y \Delta^{2} \mu_{2}{ }^{(0)} / 4\right)+\left(\epsilon^{2}+\Delta^{2}\right) x y \Delta^{2} \mu_{3}{ }^{(0)}-2 \epsilon x y \Delta^{2} \mu_{2}{ }^{(0)} \mu_{2}{ }^{(0)} \\
& +3 \epsilon x y \Delta^{2} \mu_{4}{ }^{(0)}+x y \Delta^{2} \mu_{5}{ }^{(0)}+\left[\left(\Delta^{6} / 16\right)+\left(3 \epsilon^{2} \Delta^{4} / 16\right)+2\left(\Delta^{2}+\epsilon^{2}\right) x y \Delta^{2} \mu_{2}{ }^{(0)}+6 \epsilon x y \Delta^{2} \mu_{3}{ }^{(0)}+2 x y \Delta^{2} \mu_{4}{ }^{(0)}\right] \cdot \epsilon(\mathbf{k}, j) \\
& +\left[\left(18 \epsilon \Delta^{4} / 16\right)+\left(3 \epsilon^{3} \Delta^{2} / 4\right)+9 \epsilon x y \Delta^{2} \mu_{2}{ }^{(0)}+3 x y \Delta^{2} \mu_{3}{ }^{(0)}\right] \cdot \epsilon^{2}(\mathbf{k}, j)+\left[\left(10 \Delta^{4} / 16\right)+6 \epsilon^{2} \Delta^{2}+\epsilon^{4}+4 x y \Delta^{2} \mu_{2}{ }^{(0)}\right] \cdot \epsilon^{3}(\mathbf{k}, j) \\
& +\left[\left(25 \epsilon \Delta^{2} / 4\right)+10 \epsilon^{3}\right] \cdot \epsilon^{4}(\mathbf{k}, j)+\left[\left(3 \Delta^{2} / 2\right)+15 \epsilon^{2}\right] \cdot \epsilon^{5}(\mathbf{k}, j)+7 \epsilon \cdot \epsilon^{6}(\mathbf{k}, j)+\epsilon^{7}(\mathbf{k}, j) \\
& +x^{2} y^{2} \Delta^{4}\left[\sum_{\alpha} W^{3}\left(\mathbf{R}_{\alpha}\right) \exp \left(i \mathbf{k} \cdot \mathbf{R}_{\alpha}\right)-(-1)^{j} \sum_{\beta} W^{3}\left(\mathbf{R}_{\beta}\right) \exp \left(i \mathbf{k} \cdot \mathbf{R}_{\beta}\right)\right]
\end{aligned}
$$

The moments of the over-all density-of-states function $\rho(E)$ are found by using Eq. $(7 \mathrm{c})$ :

$$
\begin{aligned}
& \mu_{0}=1 \text {, } \\
& \mu_{1}=\epsilon \text {, } \\
& \mu_{2}=\Delta^{2} / 4+\mu_{2}{ }^{(0)}, \\
& \mu_{3}=\left(\epsilon \Delta^{2} / 4\right)+3 \epsilon \mu_{2}{ }^{(0)}+\mu_{3}{ }^{(0)} \text {, } \\
& \mu_{4}=\left(\Delta^{4} / 16\right)+\left(\Delta^{2}+2 \epsilon^{2}\right) \mu_{2}{ }^{(0)}+4 \epsilon \mu_{3}{ }^{(0)}+\mu_{4}{ }^{(0)} \text {, } \\
& \mu_{\overline{5}}=\left(\epsilon \Delta^{4} / 16\right)+\left(5 \epsilon \Delta^{2} \mu_{2}{ }^{(0)} / 2\right)+\left[\left(5 \Delta^{2} / 4\right)+5 \epsilon^{2}\right] \mu_{3}{ }^{(0)}+5 \epsilon \mu_{4}{ }^{(0)}+\mu_{5}{ }^{(0)} \text {, } \\
& \mu_{6}=\left(\Delta^{6} / 64\right)+\left(\frac{9}{16} \Delta^{4}+\frac{3}{2} \epsilon^{2} \Delta^{2}+3 x y \Delta^{2} \mu_{2}{ }^{(0)}\right) \mu_{2}{ }^{(0)}+\left(\frac{9}{2} \epsilon \Delta^{2}+2 \epsilon^{3}\right) \mu_{3}{ }^{(0)}+\left(\frac{3}{2} \Delta^{2}+9 \epsilon^{2}\right) \mu_{4}{ }^{(0)}+6 \epsilon \mu_{5}{ }^{(0)}+\mu_{6}{ }^{(0)} \text {, } \\
& \mu_{7}=\left(\epsilon \Delta^{6} / 64\right)+\left[\left(21 \epsilon \Delta^{4} / 16\right)+7 \epsilon x y \Delta^{2} \mu_{2}{ }^{(0)}+7 x y \Delta^{2} \mu_{3}{ }^{(0)}\right] \mu_{2}{ }^{(0)}+\left[\frac{7}{8} \Delta^{4}+\left(21 \epsilon^{2} \Delta^{2} / 4\right)\right] \mu_{3}{ }^{(0)} \\
& +\left(7 \epsilon \Delta^{2}+7 \epsilon^{3}\right) \mu_{4}{ }^{(0)}+\left(\frac{7}{4} \Delta^{2}+14 \epsilon^{2}\right) \mu_{5}^{(0)}+7 \epsilon \mu_{6}{ }^{(0)}+\mu_{7}{ }^{(0)} .
\end{aligned}
$$


The lower moments [up to $M_{5}(\mathbf{k}, j)$ and $\mu_{5}$ ] in Eqs. (12) and (13) agree with those given by Velicky et al., using a somewhat different method which did not require any knowledge of the true Green's function. To compare with present results, we have followed the recipe of Velicky et al. and computed the next two moments, as shown in Appendix B. They too agree with Eqs. (12) and (13), as expected. These results can be regarded as a consistency check on the average Green's function for multiple-branched exciton bands derived by Hong and Robinson. This consistency indicates that the Green's function is correct. A further point to be emphasized here is that, knowing the true Green's function we can always obtain the moments. The reverse is apparently not true.

The following conclusions were reached: (1) $\Sigma(\mathbf{k}, j)$ is $\mathbf{k}$ independent and branch independent up to $Z^{-5}$. (2) Up to the 7th order, the moments of the mixed crystal density-of-states function, the $\mu_{\gamma}$ 's, are only functions of the $\mu_{n}{ }^{(0)}$ 's, the moments of the corresponding pure crystal density-of-states function. ${ }^{23}$ The significance of these conclusions will be discussed later in connection with the coherent potential approximation. Finally, we note that, although the present results are specifically for multiple-branched exciton bands with nontrivial interchange symmetries of order two, their extension to more complicated systems (such as benzene) can be easily done. We might also add that the second term (the summation over $\beta$ ) in the last bracket of Eq. (11) should be dropped if there is only one molecule per unit cell.

\section{THE CPA GREEN'S FUNCTION AND MOMENTS}

The CPA Green's function has been independently proposed by several authors, ${ }^{7-9}$ each deriving it differently. So far this is the only approximate Green's function that is amenable to either model calculations ${ }^{7,10}$ or actual applications. ${ }^{12,24}$ It has an especially simple form:

$$
\langle G(\mathbf{k}, j)\rangle_{c}=\left[E-\epsilon-\epsilon(\mathbf{k}, j)-\Sigma_{c}(E)\right]^{-1},
$$

where

$$
\Sigma_{c}(E)=C_{A} C_{B} \Delta^{2} /\left[\langle G(E)\rangle^{-1}+\left(C_{B}-C_{A}\right) \Delta+\Sigma_{c}(E)\right]
$$

is the CPA self-energy and

$$
\langle G(E)\rangle=N^{-1} \sum_{j} \sum_{k}\langle G(\mathbf{k}, j)\rangle_{c} .
$$

It was elaborated by Velicky et $a l .^{10}$ that this is essentially a self-consistent single-site approximation where interference effects due to scattering are neglected ${ }^{7}$ Since Velicky et al..$^{10}$ made no references to the exact form of the true self-energy [Eq. (2)], their arguments seem more "physical" and less "analytical" than those presented by Onodera and Toyozawa. ${ }^{7}$ The latter authors actually expanded the CPA self-energy and compared the expansion with the true self-energy. At least for the first few terms, they were able to show that, if the interference scattering events were first treated statistically in an exact manner and then replaced with noninterference terms, the CPA self-energy was obtained. In this section, we shall perform the expansion of $\Sigma_{c}(E)$ in a systematic way with the aid of a generating function. After some discussions on the moments based on the CPA Green's function, we shall take up in the next section the relationship between the scattering events included in the exact self-energy and those in the CPA self-energy.

Expansion of $\Sigma_{c}(E)$ in terms of $\langle G(E)\rangle$ can be achieved by first solving the quadratic equation [Eq. (15)]. The only physically significant root is:

$$
\begin{aligned}
\Sigma_{c}(E)=\frac{1}{2}\left[-\langle G(E)\rangle^{-1}(1+\omega s)\right. \\
\left.+\langle G(E)\rangle^{-1}\left(1+2 \omega s+s^{2}\right)^{1 / 2}\right]
\end{aligned}
$$

For convenience, we have introduced new parameters: $\omega \equiv C_{B}-C_{A}$ and $s \equiv \Delta\langle G(E)\rangle$. The second term has the form of a generating function. This suggests that we define

$$
u(\omega, s) \equiv 2^{-1}\left(1+2 \omega s+s^{2}\right)^{1 / 2} \equiv \sum_{l=0}^{\infty} q_{l}(\omega) s^{l} .
$$

Using the standard techniques ${ }^{25}$ in deriving recursion formula from the generating functions, we have:

$$
\begin{aligned}
& (1-\omega) q_{l}^{\prime}=-\omega l q_{l}-(l-2) q_{l-1}, \\
& (l+1) q_{l+1}=-(2 l-1) \omega q_{l}-(l-2) q_{l-1} .
\end{aligned}
$$

The close resemblances between Eq. (18) and the corresponding generating function for the Legendre polynomials suggests that the $q i$ 's might be related to the latter in a simple way. For the Legendre polynomials $L_{l}(\omega)$, we have

$$
T(\omega, s) \equiv\left(1-2 \omega s+s^{2}\right)^{-1 / 2} \equiv \sum_{l=0}^{\infty} L_{l}(\omega) s^{l},
$$

and hence,

$$
u(\omega,-s)=2^{-1} \sum_{l=0}^{\infty}\left(1-2 \omega s+s^{2}\right) L_{l}(\omega) s^{l} .
$$

By substituting Eq. (18) into Eq. (21) and comparing the coefficients on both sides, we find that

$$
\begin{aligned}
q_{l}(\omega) & =(-1)^{l}\left[L_{l}(\omega) / 2-\omega L_{l-1}(\omega)+L_{l-2}(\omega) / 2\right] \\
& =(4 l-2)^{-1}\left[(-1)^{l+1} L_{l}(\omega)+(-1)^{l} L_{l-2}(\omega)\right] .
\end{aligned}
$$

We have used the recursion formula ${ }^{25}$ for Legendre polynomials in the last step. From the known properties of the Legendre polynomials, it is apparent that in general the $q_{l}$ are not orthonormal; however, they 
are either even functions or odd functions, just like the Legendre polynomials.

Some of the lower $q_{l}$ 's can be easily obtained:

$$
\begin{aligned}
& q_{0}=1 / 2, \\
& q_{1}=\omega / 2, \\
& q_{2}=\frac{1}{4}\left(1-\omega^{2}\right), \\
& q_{3}=\frac{1}{4}\left(-\omega+\omega^{3}\right), \\
& q_{4}=\frac{1}{16}\left(-1+6 \omega^{2}-5 \omega^{4}\right), \\
& q_{5}=\frac{1}{16}\left(3 \omega-10 \omega^{3}+7 \omega^{5}\right), \\
& q_{6}=\frac{1}{32}\left(1-15 \omega^{2}+35 \omega^{4}-21 \omega^{6}\right), \\
& q_{7}=\frac{1}{32}\left(-5 \omega+35 \omega^{3}-63 \omega^{5}+33 \omega^{7}\right) .
\end{aligned}
$$

The expansion of $\Sigma_{c}(E)$ is now a simple matter. From Eqs. (17) and (18), we have

$$
\Sigma_{c}(E)=\langle G(E)\rangle^{-1} \sum_{l=2}^{\infty} q_{l}(\omega) s^{l}=\sum_{l=2}^{\infty} q_{l}(\omega) \Delta^{l}\langle G(E)\rangle^{l-1} .
$$

This can be represented diagrammatically as shown in Fig. 1(b). To distinguish from Fig. 1(a), we have used a cross $(+)$ instead of an $X$. Each cross is now associated with $q_{l}(\omega)$ rather than $Y_{l}(\omega)$. The relationship between $q_{l}(\omega)$ and $Y_{l}(\omega)$ will be taken up in the next section. Note that $q_{2}(\omega)$ and $q_{3}(\omega)$ are identical to $Y_{2}(\omega)$ and $Y_{3}(\omega)$. The CPA Green's function is now represented as a double line to distinguish from the true Green's function represented as a thick line in Fig. 1(a).

Similar to Eq. (10), we now expand the CPA selfenergy in powers of $Z^{-1}\left(Z \equiv E-i 0^{+}\right)$:

$$
\Sigma_{c}(E) \equiv \sum_{p=1}^{\infty} \Lambda_{p}^{0} Z^{-p}
$$

Using the same techniques used in the last section, the $\Lambda_{p}{ }^{0}$ can be determined from equations similar to Eqs. (8) and (9), except that the exact Green's function $\langle G(\mathbf{k}, j)\rangle[$ defined in Eq. (3)] is now replaced by the CPA Green's function $\langle G(\mathbf{k}, j)\rangle_{c}$ [defined in Eq. (14)]. It is found that the first few coefficients are identical to Eq. (11) without the $\mathbf{k}$ - and $j$-dependent terms:

$$
\begin{gathered}
\Sigma_{c}(E)=x y \Delta^{2}\left[Z^{-1}-\epsilon Z^{-2}+\left(\mu_{2}{ }^{(0)}+\epsilon^{2}\right) Z^{-3}\right. \\
+\left(\mu_{3}{ }^{(0)}-\epsilon^{3}-\epsilon \mu_{2}{ }^{(0)}\right) Z^{-4}+\left(\mu_{4}{ }^{(0)}+2 \epsilon^{2} \mu_{2}{ }^{(0)}+\epsilon^{4}\right. \\
\left.+x y \Delta^{2} \mu_{2}{ }^{(0)}\right) Z^{-5}+\left(\mu_{5}{ }^{(0)}-\epsilon^{5}+\epsilon \mu_{4}{ }^{(0)}+\frac{1}{2} \Delta^{2} \mu_{3}{ }^{(0)}-\epsilon^{3} \mu_{3}{ }^{(0)}\right. \\
\left.\left.-\frac{1}{4} \epsilon \Delta^{2} \mu_{2}{ }^{(0)}-2 \epsilon \mu_{2}{ }^{(0)} \mu_{2}{ }^{(0)}\right) Z^{-6}+\cdots\right] .
\end{gathered}
$$

Notice that we have again put $\frac{1}{2}\left(\boldsymbol{\epsilon}_{A}+\boldsymbol{\epsilon}_{B}\right) \equiv 0$, and $C_{A} \equiv x, C_{B} \equiv y$, and also $\epsilon \equiv \Delta \omega / 2$. The corresponding moments of $a(\mathbf{k}, j)$ and $\rho(E)$ determined from $\Sigma_{c}(E)$ are easily shown to be identical to Eqs. (12) and (13) except that the last term for $M_{7}(\mathbf{k}, j)$ in Eq. (12) is absent in the new expression for the CPA. In other words, the over-all density-of-states function determined from CPA yields the correct first eight moments (up to $\mu_{7}$ ). The corresponding spectral density will yield correctly only the first seven moments [up to $\left.M_{6}(\mathbf{k}, j)\right]$. In the numerical calculations of Hong and Robinson, ${ }^{12}$ only the first two moments were checked. In a later publication from this laboratory, ${ }^{15}$ we shall assess the quality of our numerical calculations by comparing them with these higher moments.

Finally, we should point out that the conclusions reached here are consistent with the fact that the CPA is a self-consistent theory, i.e., it is the best theory possible based on: (1) the pure crystal density-of-states function and (2) the energy of the $\mathbf{k}, j$ state in the pure crystal [i.e., $\boldsymbol{\epsilon}(\mathbf{k}, j)]$. As we have seen, as long as the mixed crystal properties [e.g., $M_{p}(\mathbf{k}, j)$ and $\mu_{p}$ discussed here] can be determined solely from these two parameters, the CPA always yields the correct answers. The failure of the CPA to yield the right $M_{7}(\mathbf{k}, j), \mu_{8}$, and higher moments is apparently due to the fact that the complete dispersion relation is now called for to evaluate these quantities.

\section{THE EXACT GREEN'S FUNCTION AND THE CPA GREEN'S FUNCTION}

To probe into the physical meanings of the CPA, we first examine the third and the fourth diagrams in Fig. 1(a). It was mentioned before that the $\mathbf{k}, j$-dependent term in Eq. (11) actually came from the fourth diagram. If we expand the 3 rd and the 4 th terms and examine terms up to $Z^{-6}$ power, we find that

$$
\begin{aligned}
\Delta^{4} Y_{4}(\omega)\left\{\sum_{j^{\prime}} \sum_{j^{\prime \prime}} \sum_{j^{\prime \prime \prime}} \sum_{k^{\prime}} \sum_{k^{\prime \prime}} \sum_{k^{\prime \prime \prime}} N^{-3}\left\langle G\left(\mathbf{k}^{\prime}, j^{\prime}\right)\right\rangle\left\langle G\left(\mathbf{k}^{\prime \prime}, j^{\prime \prime}\right)\right\rangle\left\langle G\left(\mathbf{k}^{\prime \prime \prime}, j^{\prime \prime \prime}\right)\right\rangle\right\} \\
\quad=\Delta^{4} Y_{4}(\omega)\left\{\cdots+\left(9 \epsilon x y \Delta^{2}+3 \mu_{3}{ }^{(0)}+15 \epsilon \mu_{2}{ }^{(0)}+10 \epsilon^{3}\right) Z^{-6}+\cdots\right\},
\end{aligned}
$$

whereas

$$
\begin{aligned}
\Delta^{4} Y_{2}^{2}(\omega)\left\{\sum_{j^{\prime}} \sum_{j^{\prime \prime}} \sum_{j^{\prime \prime \prime}} \sum_{k^{\prime}} \sum_{k^{\prime \prime}} \sum_{k^{\prime \prime \prime}} \vec{\delta}\left(\mathbf{k}-\mathbf{k}^{\prime}+\mathbf{k}^{\prime \prime}-\mathbf{k}^{\prime \prime \prime}\right) N^{-2}\left\langle G\left(\mathbf{k}^{\prime}, j\right)\right\rangle\left\langle G\left(\mathbf{k}^{\prime \prime}, j^{\prime \prime}\right)\right\rangle\left\langle G\left(\mathbf{k}^{\prime \prime \prime}, j^{\prime \prime \prime}\right)\right\rangle\right\} \\
=\Delta^{4} Y_{2}{ }^{2}(\omega)\left\{\cdots+\left(9 \epsilon x y \Delta^{2}+3 \mu_{3}{ }^{(0)}+15 \epsilon \mu_{2}{ }^{(0)}+10 \boldsymbol{\epsilon}^{3}\right) Z^{-6}\right. \\
\left.+\left[\sum_{\alpha} W^{3}\left(\mathbf{R}_{\alpha}\right) \exp \left(i \mathbf{k} \cdot \mathbf{R}_{\alpha}\right)-(-1)^{j} \sum_{\beta} W^{3}\left(\mathbf{R}_{\beta}\right) \exp \left(i \mathbf{k} \cdot \mathbf{R}_{\beta}\right)\right] Z^{-6}+\cdots\right\} .
\end{aligned}
$$


The first five terms (not shown) are identical. Even the $Z^{-6}$ terms are similar except for the $\mathbf{k}, j$-dependent term. This suggests that if we replace the scattering event associated with the fourth diagram with that of the third diagram while retaining the probability function $\left[\Delta^{4} Y_{2}^{2}(\omega)\right]$, the subsequent self-energy will be $\mathbf{k}, j$ independent. That we are indeed getting the CPA selfenergy by such an operation can be further illustrated by examining the relationship between $q_{4}(\omega)$ and the linear combination of $Y_{4}(\omega)$ and $Y_{2}^{2}(\omega)$. If we write

$$
q_{4}(\omega)=C_{1} Y_{4}(\omega)+C_{2} Y_{2}^{2}(\omega)
$$

simultaneous equations for $C_{1}$ and $C_{2}$ can be obtained by comparing powers of $\omega$ on both sides. The solution in this particular instance is simply $C_{1}=1, C_{2}=1$. We can illustrate the operation of replacing the braced term (expressing the scattering event) in Eq. (28) by that in Eq. (27) schematically as shown in the first row of Fig. 2. The fourth diagram is now replaced by the last one in the first row of Fig. 2. The dotted line connecting the two crosses indicates the collapse of the correlation, and hence the collapse of interference, between the two impurities.

Notice that Eq. (29) is easily soluble because of our particular choice of parameter $(\omega)$. If the $P_{i}$ 's were written in the form suggested by Yonezawa and Matsubara, ${ }^{6}$ the solutions would not be so apparent. Furthermore, because of the dual symmetry $y^{6,7,12}$ of the problem, $\omega$ appears to be a more natural parameter.

Higher-order terms can be treated in a manner similar to the one above. In each case, simultaneous equations have been obtained and solved. Some of the results are shown schematically in Fig. 2. Notice that the coefficients obtained in this way do indeed predict the correct total number of distinct diagrams becoming indistinguishable in the CPA. For example, in the second row, the last five diagrams have actually been converted from those of Fig. 3. The first four in Fig. 3

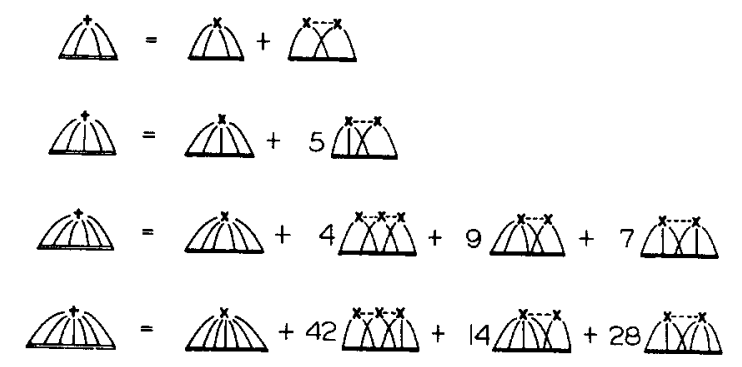

FIG. 2. Diagrammatic representation of the process through which the CPA self-energy $\Sigma_{c}(E)$ is obtained from the exact self-energy $\Sigma(\mathbf{k}, j)$. Diagrams on the rhs which are due to the interference scattering are replaced by diagrams due to noninterference scattering to obtain the CPA diagrams on the lhs. This process is represented diagrammatically here by joining the vertices with dotted lines. The coefficient in front of a diagram shows the total number of diagrams belonging to the particular category.

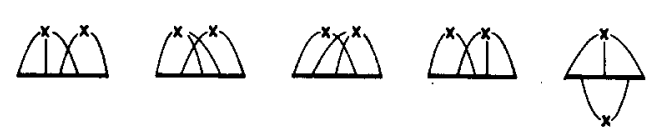

FIG. 3. Diagrams due to the interference scattering which are included as the last term on the rhs of the second row in Fig. 2.

are $\mathbf{k}, j$ dependent $t^{6,20}$ and identical, whereas the fifth one is not. However, in the CPA, they are all replaced by the same diagram as indicated in Fig. 2. Alternatively, we can write:

$$
q_{5}(\omega)=Y_{5}(\omega)+5 Y_{2}(\omega) \cdot Y_{3}(\omega)
$$

The analysis presented here further supports the original conjectures by Onodera and Toyozawa ${ }^{7}$ concerning the significance of the CPA. A more systematic method of examining successive terms in both the expansions is made possible by the conversion of the parameter $C_{B}$ to $\omega$. In Fig. 2, the expansion terms of $\sum_{c}(E)$ are examined up to the sixth power of $\langle G(E)\rangle$. In each instance, a one-to-one correspondence between the diagrams included in the CPA and the exact selfenergies has been found. Although this correspondence has only been demonstrated for some terms but not proven, it is inconceivable that a more rigorous treatment ${ }^{26}$ would indicate otherwise. It has been pointed out by Yonezawa and Matsubara ${ }^{6}$ that failure to treat the statistics correctly [e.g., by putting $\left.P_{n}\left(C_{B}\right) \rightarrow C_{B}{ }^{n}\right]$ could lead to erroneous results. The success of the CPA indicates that one should instead follow another approach, namely that of treating the statistics correctly first and then removing the interference terms.

\section{CONCLUSION}

In this paper, we emphasize the important role of the exact self-energy. Its expression is necessarily complicated because of the intrinsic complexity of the problem. Although the direct application to a real system seems rather elusive at the moment, nevertheless, important information can be extracted from it. Not only the moments can be readily evaluated from it but also the CPA self-energy comes naturally from it if certain terms are modified. For later uses, we have also computed explicitly some lower moments of the mixedcrystal spectral density and the over-all density-ofstates function. It has also been shown that the CPA self-energy yields the correct lowest eight moments for the over-all density-of-states function.

\section{APPENDIX A: POLYNOMIALS USED IN THE EXPANSION OF THE EXACT SELF-ENERGY}

The polynomials $P_{l}$ associated with the expansion of the true self-energy in terms of propagators were given 
by Yonezawa and Matsubara ${ }^{6}$ in the following form:

$$
\log \left(1-C_{B}+C_{B} e^{t}\right)=\sum_{l=1}^{\infty} \frac{P_{l}\left(C_{B}\right) t^{l}}{l !} .
$$

These polynomials can be conveniently converted into functions of $\omega\left(=C_{B}-C_{A}\right)$ by simply rewriting $\mathrm{Eq}$. (A1) as

$$
\log \left\{\frac{1}{2}\left[1-\omega+(1+\omega) e^{t}\right]\right\}=\sum_{l=1}^{\infty} \frac{Y_{l}(\omega) t^{l}}{l !} .
$$

Recursion formula can be obtained by partial differentiations of Eq. (A2) with respect to $\omega$ and $t$. We have found that

with

$$
Y_{l}(\omega)=\frac{1}{2}\left(1-\omega^{2}\right) Y_{l-1}^{\prime}(\omega),
$$

$$
Y_{1}(\omega)=(1+\omega) / 2 \text {. }
$$

The first few functions are:

$$
\begin{aligned}
& Y_{1}(\omega)=(1+\omega) / 2, \\
& Y_{2}(\omega)=\left(1-\omega^{2}\right) / 4, \\
& Y_{3}(\omega)=-\left(\omega+\omega^{3}\right) / 4, \\
& Y_{4}(\omega)=\frac{1}{8}\left(-1+4 \omega^{2}-3 \omega^{4}\right), \\
& Y_{5}(\omega)=\frac{1}{8}\left(4 \omega-10 \omega^{3}+6 \omega^{5}\right), \\
& Y_{6}(\omega)=\frac{1}{16}\left(4-34 \omega^{2}+60 \omega^{4}-30 \omega^{6}\right), \\
& Y_{7}(\omega)=\frac{1}{16}\left(-34 \omega+154 \omega^{3}-210 \omega^{5}+90 \omega^{7}\right) .
\end{aligned}
$$

Notice that

and

$$
Y_{2}(\omega)=q_{2}(\omega)
$$

$$
Y_{3}(\omega)=q_{3}(\omega) .
$$

\section{APPENDIX B: COMPUTATION OF $\left\langle H^{6}\right\rangle,\left\langle H^{7}\right\rangle$, AND MOMENTS}

In the text, various moments $M_{p}(\mathbf{k}, j)$ and $\mu_{p}(E)$ have been obtained from an expansion of the true Green's function. An alternative way would be to evaluate them directly from the mixed-crystal Hamiltonian in a localized representation. Using the notations by Velicky et al., ${ }^{10}$ we can write

$$
H=D+W
$$

where $D$ contains all the diagonal elements (taking up values of either $\epsilon_{A}$ of $\epsilon_{B}$, randomly) and $W$ contains all the off-diagonal elements and is the same as in the pure crystal. To get $M_{p}(\mathbf{k}, j)$ and $\mu_{p}(E)$, we have to calculate $\left\langle H^{p}\right\rangle$, i.e., the $p$ th power of the matrix $H$ averaged over all the different configurations of guest-host distributions. $\left\langle H^{p}\right\rangle$ can be calculated from the relation:

$$
\left\langle H^{p}\right\rangle=\left\langle(D+W)^{p}\right\rangle=\left\langle D^{p}\right\rangle+\left\langle D^{p-1} W\right\rangle+\cdots+\left\langle W^{p}\right\rangle .
$$

The $\left\langle H^{0}\right\rangle$ to $\left\langle H^{5}\right\rangle$ are given in the paper by Velicky et al. The $\left\langle H^{6}\right\rangle$ and $\left\langle H^{7}\right\rangle$ have been calculated by us and are as follows:

$$
\begin{aligned}
& \left\langle H^{6}\right\rangle=\left(\Delta^{6} / 64\right)+x y \Delta^{2} \mu_{4}{ }^{(0)}+2 \epsilon x y \Delta^{2} \mu_{3}{ }^{(0)}+{ }_{4}^{3} x y \Delta^{4} \mu_{2}{ }^{(0)}+\left({ }_{8}^{3} \epsilon \Delta^{4}+4 \epsilon x y \Delta^{2} \mu_{2}{ }^{(0)}+2 x y \Delta^{2} \mu_{3}{ }^{(0)}\right) W \\
& +\left(\frac{3}{8} \Delta^{4}+\frac{9}{4} \epsilon^{2} \Delta^{2}+3 x y \Delta^{2} \mu_{2}^{(0)}\right) W^{2}+\left(4 \epsilon \Delta^{2}+4 \epsilon^{3}\right) W^{3}+\left(\frac{5}{4} \Delta^{2}+10 \epsilon^{2}\right) W^{4}+6 \epsilon W^{5}+W^{6}, \\
& \left\langle H^{7}\right\rangle=\left(\epsilon \Delta^{6} / 64\right)+{ }_{4}^{3} \epsilon \cdot y y \Delta^{2} \mu_{2}{ }^{(0)}+\left(\epsilon^{2}+\Delta^{2}\right) x y \Delta^{2} \mu_{3}{ }^{(0)}-2 \epsilon x y \Delta^{2} \mu_{2}{ }^{(0)} \mu_{2}{ }^{(0)}+3 \epsilon x y \Delta^{2} \mu_{4}{ }^{(0)}+x y \Delta^{2} \mu_{5}{ }^{(0)} \\
& +\left[\left(\Delta^{6} / 16\right)+\left(3 \epsilon^{2} \Delta^{4} / 16\right)+2\left(\Delta^{2}+\epsilon^{2}\right) x y \Delta^{2} \mu_{2}{ }^{(0)}+6 \epsilon x y \Delta^{2} \mu_{3}{ }^{(0)}+2 x y \Delta^{2} \mu_{4}{ }^{(0)}\right] W \\
& +\left[\left(18 \epsilon \Delta^{4} / 16\right)+{ }_{4}^{3} \epsilon^{2} \Delta^{2}+9 \epsilon x y \Delta^{2} \mu_{2}{ }^{(0)}+3 x y \Delta^{2} \mu_{3}{ }^{(0)}\right] W^{2}+\left[\left(10 \Delta^{4} / 16\right)+6 \epsilon^{2} \Delta^{2}+\epsilon^{4}+4 x y \Delta^{2} \mu_{2}{ }^{(0)}\right] W^{3} \\
& +\left[\left(\frac{25}{4} \epsilon \Delta^{2}\right)+10 \epsilon^{3}\right] W^{4}+\left(\frac{3}{2} \Delta^{2}+15 \epsilon^{2}\right) W^{5}+7 \epsilon W^{6}+W^{7}+x^{2} y^{2} \Delta^{4} W^{(3)} \text {. }
\end{aligned}
$$

The notation and conventions are the same as those of Velicky et al. except that $\Delta=-\delta$. The latter distinction is not very important since only even powers are involved. The expression of $\left\langle H^{7}\right\rangle$ involves the matrix $W^{(3)}$ defined as

$$
W_{i j}^{(3)}=W_{i j}{ }^{3} \text {. }
$$

It is obvious that $W^{(3)} \neq W^{3}$. Ultimately, this is the term that is responsible for the $\mathbf{k}, j$-dependent term in Eq. (11). Some of the more cumbersome terms in Eq. (B1) are given in the following:

$$
\begin{aligned}
\langle D W D W D D\rangle & =\epsilon^{2} \Delta^{2} W^{2} / 4 \\
\langle D D W D W D\rangle & =\epsilon^{2} \Delta^{2} W^{2} / 4 \\
\langle D W D D W D\rangle & =\epsilon^{2} \Delta^{2} W^{2} / 4+x y \Delta^{4} \mu_{2}{ }^{(0)} / 4 \\
\langle D W D W D W D\rangle & =\epsilon^{4} W^{3}+2 \epsilon^{2} x y \Delta^{2} \mu_{2}{ }^{(0)} W+\epsilon^{2} x y \Delta^{2} \mu_{3}{ }^{(0)}+x^{2} y^{2} \Delta^{4} W^{(3)}, \\
\langle D D W D W W D\rangle & =\left(\epsilon^{2} \Delta^{2} W^{3} / 4\right)+\left(x y \Delta^{4} \mu_{2}{ }^{(0)} W / 4\right) \\
\langle D W D D W W D\rangle & =\left(\epsilon^{2} \Delta^{2} W^{3} / 4\right)+\left(x y \Delta^{4} \mu_{3}{ }^{(0)} / 4\right) \\
\langle D W W D W W D\rangle & =\epsilon^{3} W^{4}+\epsilon x y \Delta^{2}\left(2 \mu_{2}{ }^{(0)} W^{2}+\mu_{4}{ }^{(0)}-2 \mu_{2}{ }^{(0)} \mu_{2}{ }^{(0)}\right) \\
\langle D W D W D W W\rangle & =\epsilon^{3} W^{4}+\epsilon x y \Delta^{2} \mu_{2}{ }^{(0)} W^{2} \\
\langle D W D W W D W\rangle & =\epsilon^{3} W^{4}+\epsilon x y \Delta^{2}\left(\mu_{2}{ }^{(0)} W^{2}+\mu_{3}{ }^{(0)} W\right), \\
\langle D W D W W W D\rangle & =\epsilon^{3} W^{4}+\epsilon x y \Delta^{2}\left(\mu_{3}{ }^{(0)} W+\mu_{4}{ }^{(0)}\right), \text { etc. }
\end{aligned}
$$


In deriving these, we have used the relationship

$$
\frac{1}{4} \Delta^{2}=\epsilon^{2}+x y \Delta^{2}
$$

As pointed out by Velicky et al.,$^{10}$ the expressions for the $M_{p}(\mathbf{k}, j)$ 's have the same structure as those of the $\left\langle H^{p}\right\rangle$ 's and can be easily obtained by a transformation to the delocalized (Bloch) representation. As expected, they are identical to Eq. (12) which was obtained in a somewhat different manner. The $\mu_{p}$ 's can be obtained again by summation of $M_{p}(\mathbf{k}, j)$ over $\mathbf{k}$ and $j$.

* Supported by NSF Grant GP-18718 and NIH Grant NS08116.

${ }^{1}$ For general review and bibliographies, see N. F. Mott, Advan. Phys. 16, 49 (1967); N. F. Mott and W. D. Twose, ibid. 10, 107 (1961); J. Hori, Spectral Properties of Disordered Chains and Lattices (Pergamon, Oxford, England, 1968).

${ }^{2}$ C. Domb, A. A. Maradudin, E. W. Montroll, and G. H. Weiss, Phys. Rev. 115, 18, 24 (1959).

${ }^{3}$ P. Dean, Proc. Phys. Soc. (London) 73, 413 (1959); Proc. Roy. Soc. (London) A254, 507 (1960); A260, 263 (1961); P. Dean and J. L. Martin, ibid. A259, 409 (1960); P. Dean and M. D. Bacon, ibid. A283, 64 (1965); D. N. Payton III and W. M. Visscher, Phys. Rev. 154, 802 (1967).

${ }^{4}$ J. Hori, Progr. Theoret. Phys. (Kyoto) 18, 367 (1957), 31, 940 (1964); J. Hori and T. Asahi, ibid. 17, 523 (1957).

5 M. Lax, Rev. Mod. Phys. 23, 287 (1951); Phys. Rev. 85, $621(1952)$.

${ }_{6}^{6}$ F. Yonezawa and T. Matsubara, Progr. Theoret. Phys. (Kyoto) 35, 357 (1966) ; 37, 1346 (1967).

${ }^{7}$ Y. Onodera and Y. Toyozawa, J. Phys. Soc. Japan 24, 341 (1968).

${ }^{8}$ D. W. Taylor, Phys. Rev. 156, 1017 (1967).

${ }^{9}$ P. Soven, Phys. Rev. 156, 809 (1967).

${ }^{10}$ B. Velicky, S. Kirkpatrick, and H. Ehrenreich, Phys. Rev. 175,747 (1968).

${ }_{11}$ P. Soven, Phys. Rev. 178, 1136 (1969).

12 (a) H. K. Hong and G. W. Robinson, J. Chem. Phys. 52, 825 (1970); (b) 54,1369 (1971).

is S. D. Colson, R. Kopelman, and G. W. Robinson, J. Chem. Phys. 47, 27 (1967).

${ }_{14}$ S. D. Colson, D. M. Hanson, R. Kopelman, and G. W. Robinson, J. Chem. Phys. 48, 2215 (1968).

${ }^{15}$ H. K. Hong and R. Kopelman, "Random Lattice Calculations on Frenkel Excitons in Disordered Molecular Crystals - ${ }^{-} B_{2} z$ Naphthalene," J. Chem. Phys. (to be published).

${ }^{16}$ See Refs. 3 and 15 and also some recent calculations by P. S. Julienne and S.-I. Choi, J. Chem. Phys. 53, 2726 (1970).
${ }_{17}$ R. Kopelman, J. Chem. Phys. 47, 2631 (1967).

${ }_{18}$ Normally, the interchange operations can only be applied to some particular $\mathbf{k}$ states $^{17}$ (see also Ref. 19). The spirit of the restricted Frenkel exciton limit ${ }^{13}$ is to neglect some small interactions so that the interchange operations can be applied to all the $\mathbf{k}$ states. Of course, use of this limit can only be made under careful scrutiny. See R. Kopelman and J. Laufer, Natl. Bur. Std. (U.S.) Spec. Publ. 323 (1970).

${ }^{19}$ (a) D. P. Craig and S. H. Walmsley, Physics and Chemistry of the Organic Solid State, edited by D. Fox, M. M. Labes, and A. Weissberger (Interscience, New York, 1963), Vol. 1, Chap. 10; (b) S. A. Rice and J. Jortner Physics and Chemistry of the Organic Solid State, edited by D. Fox, M. M. Labes, and A. Weissberger (Interscience, New York, 1967), Vol. 3, Chap. 4.

${ }^{20}$ H. K. Hong and R. Kopelman, Phys. Rev. Letters 25, 1030 (1970) and J. Chem. Phys. 55, 724 (1971).

2t For an introduction to the diagrammatic method in Green's function formalism, see R. D. Mattuck, A Guide to Feynman Diagrams in Many-body problems (McGraw-Hill, New York, 1967).

${ }^{22}$ D. Fox, J. Chem. Phys. 54, 2132 (1971).

${ }^{23}$ Notice that in Eq. (12) the last term in the expression for $M_{7}(\mathbf{k}, j)$ drops after summation over all $\mathbf{k}, j$, to get $\mu_{7}$. If $\mu_{8}$ were calculated, a term of the following form would be obtained:

$$
\mu_{8}=\cdots+\text { const }\left[\sum_{\alpha} W^{4}\left(\mathbf{R}_{\alpha}\right)+\sum_{\beta} W^{4}\left(\mathbf{R}_{\beta}\right)\right],
$$

which cannot be expressed in terms of the $\mu_{n}{ }^{(0)}$.

${ }^{24}$ S. Kirkpatrick, B. Velicky, and H. Ehrenreich, Phys. Rev. B 1, 3250 (1970); G. M. Stocks, R. W. Williams, and J. S. Faulkner, Phys. Rev. Letters 26, 253 (1971).

${ }^{25}$ See, e.g., L. I. Schiff, Quantum Mechanics (McGraw-Hill, New York, 1968), 3rd ed.

${ }^{26}$ This might involve some applications of graph theory. Note added in proof: A related paper has just comc to our attention: F. Yonezawa, Progr. 'Theoret. Phys. (Kyoto) 40, 734 (1968).

\title{
Use of the CNDO Method in Spectroscopy. V. Spin-Orbit Coupling*
}

\author{
R. L. Elims, $\dagger$ R. SQuire, and H. H. Jaffe \\ Department of Chemistry, University of Cincinnati, Cincinnati, Olio 45221
}

(Received 25 March 1971)

\begin{abstract}
We report here the use of $\mathrm{CNDO} / \mathrm{S}$ wavefunctions in the calculation of oscillator strengths of singlettriplet transitions and the corresponding triplet radiative lifetimes induced by spin-orbit coupling. The starting functions for the perturbation calculations are ground and excited state functions including limited configuration interaction. Numerical calculations are carried through for formaldehyde and azulene. The importance of the calculated $a \rightarrow \pi^{*}$ states is discussed.
\end{abstract}

In the past 30 years a great deal of work has been done to explain the strong afterglow exhibited by many organic molecules after excitation with ultraviolet light. Lewis and Kasha ${ }^{1}$ did much to advance the concept that this afterglow involved an electronic transition from the lowest triplet state to the ground state. They term this behavior phosphorescence. McClure was the first to explain the phosphorescence lifetimes of aromatic hydrocarbons on the basis of spin-orbit coupling, soc. ${ }^{2}$
The existence of soc in organic molecules has been shown to be responsible for intersystem crossing as well as phosphorescence. The effect of soc in organic systems is small enough to be treated by the RayleighSchrödinger perturbation treatment. ${ }^{3}$ The only perturbations required for the evaluation of the transition moment of a singlet-triplet transition are the perturbation of the ground state by the various triplet states and the perturbation of the lowest triplet state by the various singlet states. ${ }^{4}$ For the purpose at hand the 